\title{
Optimal capacitor placement in radial distribution systems using a fuzzy-dragonfly method
}

\author{
Essam A. Al-Ammar ${ }^{\mathrm{a} *}$, Ghazi A. Ghazi ${ }^{\mathrm{b}}$, Wonsuk $\mathrm{Ko}^{\mathrm{c}}$ \\ ${ }^{a, b, \& c}$ College of Engineering, King Saud University, Riyadh,11461,Saudi Arabia
}

\begin{abstract}
In distribution systems, supplying a reactive power along the distribution lines is essential in order to bring benefits such as power loss reduction, the voltage profile improvement, and maximizing cost saving. Shunt capacitors have been used for this purpose but the scope of these benefits depends on an appropriate location, and size of these capacitors. This paper proposes a hybrid technique consist of a Fuzzy Expert System (FES) and Dragonfly Algorithm (DA) methods to determine capacitors placement and their sizing in radial distribution system level of smart grids. The fuzzy expert system is utilized to determine the suitable locations placement of capacitors and dragonfly algorithm is utilized to find their size. This proposed method is tested at IEEE 69-bus distribution system. The obtained simulation results are compared with an existing optimization algorithm.
\end{abstract}

Keywords: Dragonfly algorithm, distribution system, fuzzy expert system, Hybrid technique, power loss, shunt capacitors

\section{Introduction}

The final stage of delivery of electricity form transmission system to consumers is distribution system in which the majority of the power losses happen because of flowing heavy currents in distribution lines. Studies on power systems showed that relatively $13 \%$ of total power generated is wasted in the form of losses at the distribution system [1]. This percentage of distribution power loss can be minimized and a voltage profile can be maintained within permissible range limits through installation of shunt capacitors. The extend of these benefits relies on the proper location, and size of these capacitors.

\subsection{Related literature}

Many hybrid techniques have been suggested for solving the capacitor placement problem. B. Souza et al.[2] introduced a genetic algorithm in conjunction with fuzzy logic. The objective function was maximizing cost savings. The proposed method is tested on a 34-bus system and the results are compared to the solution given by another search technique. B. Venkatesh et al. in [3] have proposed an evolutionary programming algorithm (EPA) that handles the problems of sitting and sizing of new shunt capacitors simultaneously while considering transformer taps, existing reactive-power sources and reconfiguration options. A fuzzy model of the objective function is developed for optimization in the EP framework. The proposed fuzzy-EPA method is tested on a 69-bus radial distribution system.

Y. Hsiao et al. [4] proposed a GA-fuzzy (GAF) approach for solving the discrete optimization problem of fixed shunt capacitor placement and sizing in the existence of voltage and current harmonics. A. Saric et al. [5] used a GA fuzzy as an optimization tool for real-time VVO of Serbian distribution system. In this case, fuzzy rules for power loss, voltage deviation, and degree of satisfaction were defined and then integrated into the GA environment to make it fast. M. Al-Hajri et al. [6] suggested a two-stage

\footnotetext{
* Manuscript received September 4, 2018; revised January 20, 2019.

Corresponding author. Tel.: +966554080602; E-mail address: essam@ksu.edu.sa.

doi: $10.12720 /$ sgce.8.2.116-124
} 
methodology to determine the proper size and locations of the capacitors for reactive power compensation of radial distribution systems. M. Prasanna et al. [7] used a new method of second order of a particle swarm optimization for calculating of capacitor sizing in radial distribution feeders for voltage profile improvement and reduction of power loss. The location of the buses or nodes is decided by a group of rules defined by the fuzzy system and the sizing of these capacitors is formed by the objective function to get maximum cost savings using the PSO.

P. Prasad et al. [8] have suggested a fuzzy-genetic approach. The location of the capacitors is identified using FES and the size of these capacitors is obtained using the genetic algorithm. The objective function is to maximize net savings in the distribution system. The proposed method is tested on 15 bus and 69 bus radial test systems. M. Reddy et al. in [9] present a fuzzy and particle swarm optimization technique for the placement of capacitors on the primary feeders of the radial distribution systems. The proposed method is tested on 15-bus, 34-bus, and 69-bus distribution systems. S. Kannan et al.[10] presented a fuzzy and hybrid Particle Swarm Optimization technique (HPSO). The location of the capacitors is specified by a set of rules by the FES and the size of the capacitors is obtained by HPSO. The suggested method is applied to a 34-bus test system.

S. Saranya et al. [11] proposed a fuzzy-differential evolution technique. A group of rules defined by the fuzzy expert system which is utilized to identify the proper locations of capacitors and their size is modeled by an objective function to get maximum savings using differential evolution method. The proposed method is applied to an IEEE 15-bus test system. D. Das [12] proposed a combination of fuzzy multi-objective and genetic algorithm (GA) based approach for optimal capacitor placement. A sensitivity index is used in order to identify best buses for installation of the capacitors. The multi-objective function is maximized using GA for obtaining the optimum sizing of fixed and switched capacitors. The proposed technique is applied and tested on 51-bus and 69-bus test systems.

In this paper, a new method of optimization based on the behavior of dragonflies called as dragonfly algorithm (DA) is applied to find optimum size of capacitors in a radial distribution system for the first time. From the literature survey, it is clear that application of DA has not been discussed so far to solve a capacitor placement problem. This encourages taking up this algorithm for solving the mentioned above problem. On comparison of the results obtained by another optimization algorithm, the proposed algorithm reveals that optimal size of capacitors as obtained by the new DA is more effective in power loss reduction, maintaining voltage profile and maximize the net saving cost.

The organization of this paper as follows; Section 2 gives a problem formulation. The optimal locations of the shunt capacitor are identified using a fuzzy expert system in section 3, dragonfly algorithm is used to find the optimal their sizes and explained in section 4. Results and discussions for one case study presented in section 5. Finally, the conclusion is given in section 6 .

\section{Problem Formulation}

\subsection{Objective function}

The objective function (1) is utilized to find the proper size and locations placement of the shunt capacitors. Backward and forward sweep approach is used as a power load flow tool [13]:

$P_{\text {loss }}=\sum_{q=2}^{N_{b}} \sum_{k=1}^{N_{b-1}}\left(\frac{P_{e f f / q}^{2}+Q_{e f f / q}^{2}}{V_{q}^{2}}\right) * R_{k}$

where,

$P_{l o s s}$ is total active power loss, $P_{e f f / q}$ and $Q_{e f f / q}$ are total effective active and reactive power loads beyond the bus q, $V_{q}$ is the voltage at bus q, $N_{b}$ is the number of system buses, $N_{b-1}$ is the total number of system branches, and $R_{k}$ is the resistance at branch $\mathrm{k}$ as displayed in Fig.1 


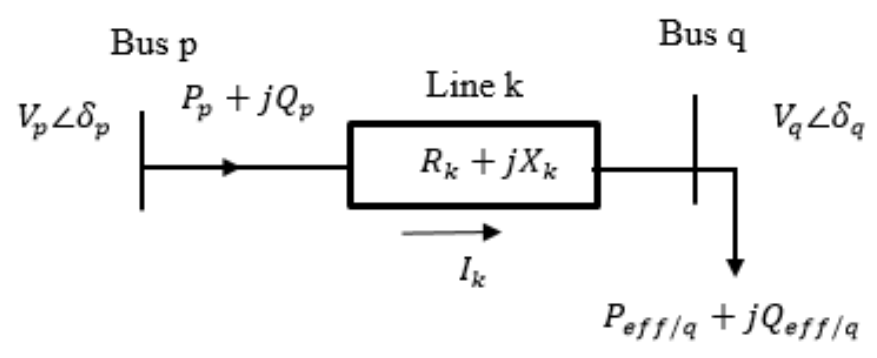

Fig. 1. Representation of two nodes in a distribution system

\subsection{The objective function constraints}

In order to find the best location and size of shunt capacitors, the objective function is subjected to the following constraints:

1) Bus voltage constraint: The voltage at each bus $\left(V_{i}\right)$ have to be within permissible minimum and maximum limits as:

$\begin{aligned} & V_{i}^{\text {min }} \leq V_{i} \leq V_{i}^{\text {max }} \\ & \text { Where, } V_{i}^{\text {min }}=0.95 V_{\mathrm{i}}^{\text {min }}=0.95 \text { and } V_{i}^{\text {max }}=1.05\end{aligned}$

2) Power flow constraint: The power flow in each line $\left(P F_{k}\right)$ must be less than the maximum limit of power flow in this line $\left(P F_{k}^{\max }\right)$ as:

$\mathrm{V}_{\mathrm{i}}^{\max }=1.05$

$$
\left|P F_{k}\right| \leq P F_{k}^{\max }
$$

3) Overall power factor constraint: The overall system power factor $\left(P_{f_{\text {overall }}}\right)$ must be greater than or equal to the minimum limit of power factor $\left(P_{f_{\text {overall }}}^{\min }\right)$ as:

$$
\left|P_{f_{\text {overall }}}\right| \geq P_{f_{\text {overall }}}^{\text {min }}
$$

4) Total reactive power constraint: The total installation of reactive power $\left(Q_{C}^{\text {Total }}\right)$ must be less than or equal the total load reactive power $\left(Q_{L}^{\text {Total }}\right)$ of the applied system as:

$$
Q_{C}^{\text {Total }} \leq Q_{L}^{\text {Total }}
$$

\subsection{Mathematical model for cost saving}

The following cost saving equation used in this paper [14]:

Here,

$$
S=K^{P}\left(\Delta P_{\text {loss }}\right)+K^{E}\left(\Delta E_{\text {loss }}\right)-K^{C} Q_{C}
$$

$\mathrm{S}$ is the saving in (\$/year), $K^{P}$ is a factor to convert the active power loss into dollars $\left(\frac{\$ 120}{k W}\right) /$ year, $K^{E}$ is a factor to convert the energy loss into dollars $\left(\frac{\$ 0.05}{k W h}\right) /$ year, $K^{C}$ is a factor to convert reactive power into dollars $\left(\frac{\$ 150}{150 \mathrm{kVAr}}\right)$ /year, $\Delta P_{\text {loss }}$ is the difference between the system power loss before and after the installation of the capacitor, $\Delta E_{\text {loss }}$ is the difference between energy loss before and after the installation of the capacitor, and $Q_{c}$ is the installed reactive power in $\mathrm{kVAr}$.

\section{Fuzzy Expert System for Capacitor Placement}

A fuzzy expert system provides a computational representation of heuristic knowledge about a specific problem. Fuzzy logic is originally proposed by L. Zadeh in 1965, it has been broadly applied in several areas of knowledge to solve mainly control and optimization problems [15]. 


\subsection{Methodology}

The inputs to the fuzzy expert system are power loss and bus voltages indices and capacitor sensitivity index (CSI) is its output. Both of power loss index and capacitor sensitivity index ranges vary from 0 to 1 p.u with five L, LM, M, HM and H triangular membership functions for each. While, bus voltage range index varies from 0.9 to $1.1 \mathrm{p}$. $\mathrm{u}$ with three $\mathrm{LM}$ and $\mathrm{H}$ trapezoidal membership functions and two LM and HM triangular membership functions as shown in Figs. 2 to 4.

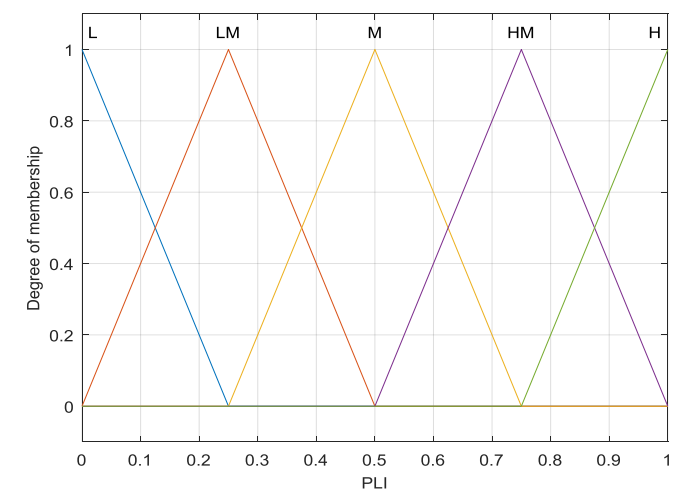

Fig. 2. Membership functions plot for power loss index

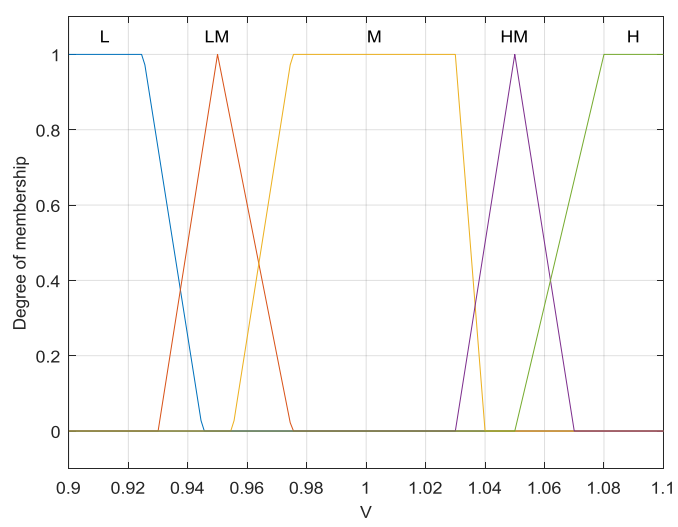

Fig. 3. Membership functions plot for voltage index

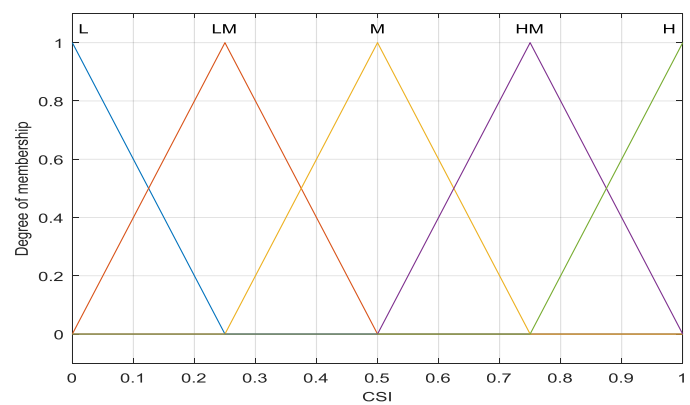

Fig. 4. Membership functions plot for capacitor sensitivity index

For the capacitor placement, rules are set to find the optimal locations of capacitor placement. These rules are represented as IF premise (antecedent), THEN conclusion (consequent). In this paper, the input 
antecedents are the bus voltages and PLI, and output consequent is the CSI. The rules are summarized in matrix form called decision matrix for CSI as given in Table 1. The L, LM, M, HM and H letters are abbreviating of Low, Low-Medium, Medium, High-Medium and High membership functions respectively. In the present work, 25 rules are constructed. For example, If PLI is Low (L) and voltage index (VI) is Low (L), then CSI is Low-Medium (LM) and so on.

Table 1. Decision matrix for CSI

\begin{tabular}{ccccccc}
\hline \multirow{2}{*}{ CSI } & & & VI & & \\
& & L & LM & M & HM & H \\
\hline L & LM & LM & L & L & L \\
& LM & M & LM & LM & L & L \\
PLI & M & HM & M & LM & L & L \\
& HM & HM & HM & M & LM & L \\
& H & H & HM & M & LM & LM \\
\hline
\end{tabular}

\subsection{Fuzzy expert system approach steps}

The steps of the FES approach explain the methodology to identify the critical busses which are more suitable for capacitor placement:

1. Read the power system data.

2. Calculate power flow using backward-forward sweep approach as a load flow.

3. Determine total active power loss.

4. By injection the self-reactive power at each bus (one by one) and perform the backward-forward sweep approach to determinate the total active power losses in each case.

5. Calculate the power loss index (PLI) using the following equation:

$$
\operatorname{PLI}(i)=\frac{L_{R}-L_{M A X}}{L_{M A X}-L_{M I N_{N}}}
$$

where, $L_{R}$ is a loss reduction, $L_{M I N}$ is a minimum reduction, $L_{M A X}$ is a maximum reduction and $\mathrm{N}$ is a number of buses.

6. The PLI and VI are the inputs to the fuzzy system.

7. The outputs of fuzzy system are defuzzified. This provides the ranking of CSI values. The buses or nodes that have the highest value of CSI are the most fit for capacitor placement.

8. Stop.

\section{Dragonfly Algorithm (DA) for Capacitor Sizing}

Dragonfly algorithm is a new swarm-based meta-heuristic optimization algorithm and it is derived based on the behavior of the dragonflies. Dragonflies are considered as small predators that hunt almost all other small insects and other marine insects and even small fishes. Swarming behavior of dragonflies is unique and rare. Dragonflies swarm for only two purposes: hunting which is called static(feeding) swarm and migration which is called dynamic (migratory) swarm. The main inspiration of the dragonfly algorithm comes from static and dynamic swarming behaviors. These behaviors are very much like the two the main phases of optimization using meta-heuristics which are exploration and exploitation.

\subsection{DA principles}

The behaviour of dragonflies swarms follows up three primitive principles [16]:

- Separation denotes to the static collision avoidance of the individuals from other individuals in the neighborhood. 
- Alignment indicates velocity matching of individuals to that of other individuals in the neighborhood.

- Cohesion represents the individuals' tendency headed for the center of the mass of the neighborhood.

\subsection{Mathematical models for DA}

The prime objective of any swarm is survival, so all of the individuals should be lured towards food sources and distracted outward enemies.

The separation is calculated as follows:

$$
S_{i}=-\sum_{j}^{N} X-X_{j}
$$

Here, $\mathrm{X}$ is the position of the current individual, $X_{j}$ refers to the position $\mathrm{j}$-th neighboring individual, and $\mathrm{N}$ is the number of neighboring individuals.

The alignment is calculated as follows:

$$
A_{i}=\frac{\sum_{j=1}^{N} V_{j}}{N}
$$

Here, $V_{j}$ shows the velocity of $\mathrm{j}$-th neighboring individual.

The cohesion is determined as follows:

$$
C_{i}=\frac{\sum_{j=1}^{N} X_{j}}{N}
$$

Here, $X_{j}$ is the position $\mathrm{j}$-th neighboring individual.

Attraction towards a food source is determined as follows:

$$
F_{i}=X^{+}-X
$$

Here, $X^{+}$refers to the position of the food source.

Distraction outwards an enemy is calculated as follows:

$$
E_{i}=X^{-}+X
$$

Here, $X^{-}$refers to the position of the enemy.

To update the position of the dragonflies in a search space and simulate their movements, two vectors are considered a step $(\Delta X)$ and position $(X)$ :

$$
\Delta X_{t+1}=\left(s S_{i}+a A_{i}+c C_{i}+f F_{i}+e E_{i}\right)+w \Delta X_{t}
$$

Here, $\mathrm{s}$ is the separation weight, $S_{i}$ is the separation of the $\mathrm{i}^{\text {-th }}$ individual, a is the alignment weight, $A_{i}$ is the alignment of $\mathrm{i}^{\text {th }}$ individual, $\mathrm{c}$ is the cohesion weight, $C_{i}$ is the cohesion of the $\mathrm{i}^{\text {th }}$ individual, $\mathrm{f}$ is the food factor, $F_{i}$ is the food source of the $\mathrm{i}^{\text {th }}$ individual, $\mathrm{e}$ is the enemy factor, $E_{i}$ is the position of enemy of the $\mathrm{i}^{\text {th }}$ individual, $\mathrm{w}$ is the inertia weight, and $\mathrm{t}$ is the iteration counter.

After calculating the step vector, the position vectors are determined as follows:

$$
X_{t+1}=X_{t}+\Delta X_{t+1}
$$

\subsection{Dragonfly algorithm steps}

The DA steps for capacitor sizing are given below:

1. Initialize the dragonflies population of search agents $X_{i}(\mathrm{i}=1,2 \ldots \mathrm{n})$.

2. Initialize step vectors $\Delta X_{i}(\mathrm{i}=1,2 \ldots \mathrm{n})$.

3. Calculate the objective function values for all dragonflies using backward forward sweep algorithm as load flow tool to determine various node voltages and active power losses. 
4. Update the food source and enemy in eqns. (7) and (8).

5. Update $\mathrm{w}, \mathrm{s}, \mathrm{a}, \mathrm{c}, \mathrm{f}$, and e values in eqn. (9).

6. Calculate S, A, C, F, and E using eqns. (4) to (8).

7. If a dragonfly has at least one neighboring dragonfly. Update velocity vector using (9) and position vector using eqn. (10).

8. Check and correct the new positions according to the boundaries of variables.

9. Repeat steps 3 to 8 until reach to maximum iteration.

10. Stop.

\section{Results and Discussion}

The proposed method is tested on IEEE 69-bus radial distributions system (RDS). Location for placement of capacitors is determined by FES approach and their sizes are evaluated using DA. The IEEE 69 bus radial distribution system consists of 1 slack bus (bus 1), 48 PQ buses and 68 branches as shown in Fig. 5. Its nominal substation transformer is rated at 4.9 MVA and $12.66 \mathrm{kV}$ [17]. Before capacitor placement (BCP), the initial power loss of this system is $225 \mathrm{~kW}$. The maximum voltage and minimum voltages are 1 and 0.9092 p.u respectively. Total annual cost of power and energy losses are $\$ 27,000$ and $\$ 98,550$ as illustrated in Table 2 .

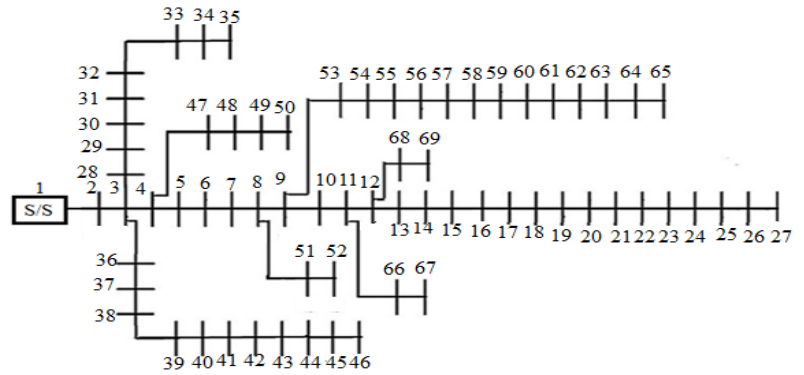

Fig. 5. Single line diagram of a 69-bus radial distribution system

After FES approach is applied for this system, it is observed that capacitor should be installed at five optimal locations i.e. buses $61,64,59,65$ and 21 , where capacitor sensitivity index value is higher as shown in Fig. 6. The size of capacitors is calculated using dragonfly algorithm and presented in Table 2. The total capacitor size injects 1.97 MVAr into the system which makes a percentage loss reduction of $42.52 \%$. The voltage constraint is attained where the maximum voltage and minimum voltages are 1.008 and 0. 0.9506 p.u as shown in Fig.7. Total annual cost of power and energy losses are $\$ 11,480$ and $\$ 41,880$ with a cost saving of $\$ 51,110$ where the benefit-cost ratio is 22.72 . The results of the proposed method for the IEEE 69 bus radial distribution system are compared with the results of particle swarm optimization with differentially perturbed velocity (PSO-DV) method in [18]. The proposed method shows that the performance and results are better when compared to the PSO-DV method.

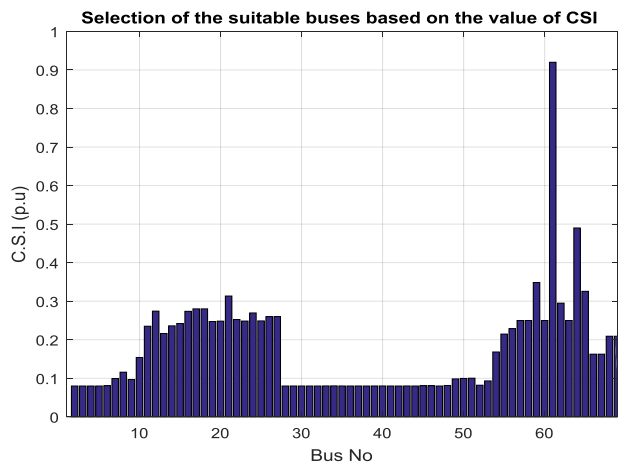

Fig. 6. Capacitor placement for 69-bus using FES 


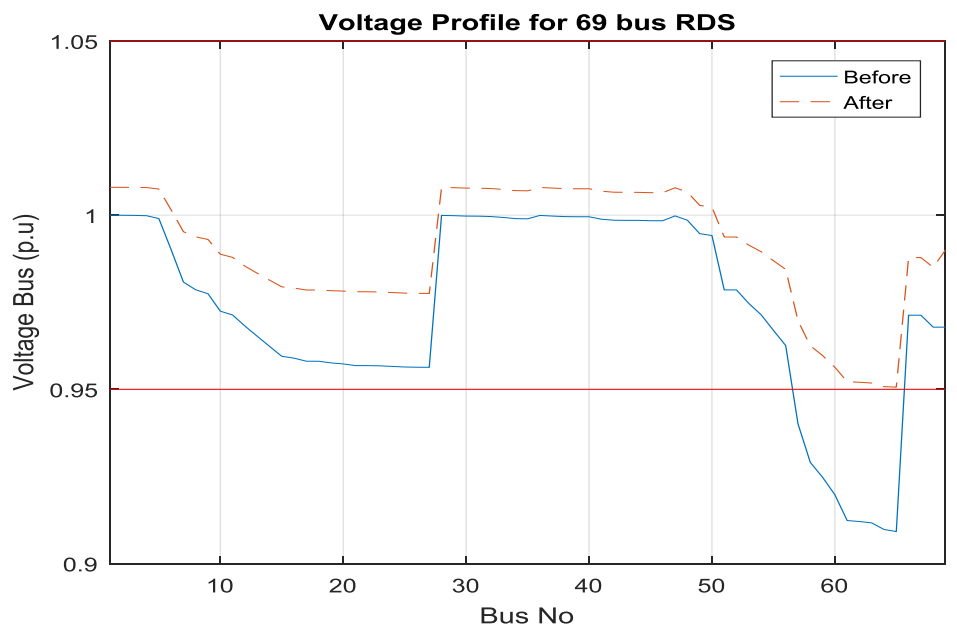

Fig. 7. Voltage profile for 69-bus before and after compensation.

Table 2. Comparison of existing and proposed method for 69-bus RDS

\begin{tabular}{cccc}
\hline Results & BCP & $\begin{array}{c}\text { PSO-DV } \\
\text { (MVAr) }\end{array}$ & $\begin{array}{c}\text { Proposed } \\
\text { In MVAr }\end{array}$ \\
\hline & 0 & $(54)$ & $(61) 1.230$ \\
& 0 & $(50)$ & $(64) 0.190$ \\
Total MVAr & 0 & $(49)$ & $(59) 0.100$ \\
Max. voltage & 1 & $(46)$ & $(65) 0.100$ \\
Min. voltage & 0.9092 & $(27)$ & $(21) 0.360$ \\
Power losses & 225 & 1.6 & 1.97 \\
Reduction $(\%)$ & 0 & 1 & 1.008 \\
Capacitor cost & 0 & 34.67 & 0.9506 \\
Power cost $\mathbf{( \$ y )}$ & 27000 & 17,640 & 129.33 \\
Energy cost & 98550 & 64,386 & 42.52 \\
Benefit $\mathbf{\$} / \mathbf{y})$ & 0 & 41,424 & 2,400 \\
Benefit-Cost & 0 & 19.73 & 51,480 \\
\hline
\end{tabular}

\section{Conclusion}

A hybrid technique consists of the fuzzy expert system and dragonfly algorithm has been presented for capacitor placement problem and tested on IEEE 69-bus radial distribution system level of smart grids to reduce the total power loss, to improve the voltage profile and to maximize the cost saving. The fuzzy expert system is used for finding the optimal locations for capacitor placement and optimal size is determined by using dragonfly algorithm method. Cost of power and energy losses, net savings, and benefit-cost ratio are also studied. The obtained results are compared with an existing optimization method; it is found that the proposed approach gives better results for the given system. 


\section{References}

[1] Song Y, Wang G, Johns A, Wang P. Distribution network reconfiguration for loss reduction using fuzzy controlled evolutionary programming. IEEE Trans. Gener., Transm., Distri., July 1997; 144(4).

[2] Souza B, Alves H, Ferreira H. Microgenetic algorithms and fuzzy logic applied to the optimal placement of capacitor banks in distribution networks. IEEE Transactions on Power Systems, May 2004; 19(2).

[3] Venkatesh B, Ranjan R. Fuzzy EP algorithm and dynamic data structure for optimal capacitor allocation in radial distribution systems, In: Proc. of IEE- Generation, Transmission and Distribution, Jan. 2006; 153(1): 80-88.

[4] Prasad P, Sivanagaraju S, Sreenivasulu N. A fuzzy-genetic algorithm for optimal capacitor placement in radial distribution systems. ARPN Journal of Engineering and Applied Sciences, June 2007; 2(3).

[5] Hsiao YT, Chen CH, Chien CC. Optimal capacitor placement in distribution systems using a combination fuzzy-GA method. International Journal of Electrical Power \& Energy Systems, 2004; 26(7): 501-508.

[6] AlHajri M, AlRashidi M, El-Hawary M. A novel discrete particle swarm optimization algorithm for optimal capacitor placement and sizing. In: Proc. of IEEE Electrical and Computer Engineering, 2007.

[7] Prasanna MB, Kannan S. Combination of fuzzy and second order PSO based capacitor placement in radial distribution feeder. In: Proc. of International Conference on Recent Trends in Computational Methods, Communication and Controls (ICON3C 2012) Proceedings published in International Journal of Computer Applications ${ }^{\circledR}(I J C A) .2012$.

[8] Sarić AT, Ćalović MS. Integrated GA-fuzzy multi-objective model for centralized Volt/Var control in distribution systems. Electric Power Components and Systems, 2005; 33(9): 1039-1055.

[9] Reddy M, Reddy V. Capacitor placement using fuzzy and particle swarm optimization method for maximum annual savings. ARPN Journal of Engineering and Applied Sciences, June 2008; 3(3).

[10] Kannan S, Renuga P, Monica A. Optimal capacitor placement and sizing using combined fuzzy-HPSO method. International Journal of Engineering, Science and Technology, 2010; 2(6): 75-84.

[11] Saranya S, Muthukumaran E, Kannan S, Kalyani S. Optimal capacitor placement in radial distribution feeders using fuzzydifferential evolution, In: Proc. of 2011 National Conference on Innovations in Emerging Technology (NCOIET), Feb. 2011.

[12] Das D. Optimum placement of shunt capacitors in a radial distribution system for substation power factor improvement using fuzzy GA method. International Journal of Electrical Power \& Energy Systems, May 2016; 77: 314-326.

[13] Abou El-Ela A, El-Sehiemy R, Kinawy A, Mouwafi1 M. Optimal capacitor placement in distribution systems for power loss reduction and voltage profile improvement. IET Generation, Transmission \& Distribution, 2016; 10(5): 1209-1221.

[14] Khalil T, Youssef H, Abdel AM, Optimal capacitor placement on radial distribution feeders in presence of nonlinear loads using binary particle swarm optimization. In: Proc. of 19th International Conference on Electricity Distribution, Vienna, 2124 May 2007.

[15] Zadeh LA. Fuzzy sets. Inf. Control, 1965; 8: 338-353.

[16] Mirjalili1 S. Dragonfly algorithm: A new meta-heuristic optimization technique for solving single-objectIve, discrete, and multi-objective problems. The Natural Computing Applications Forum, 2016.

[17] Aman M, Jasmon G, Solangi K, Bakar A, Mokhlis H. Optimum simultaneous DG and capacitor placement on the basis of minimization of power losses. International Journal of Computer and Electrical Engineering, October 2013; 5(5).

[18] Kaur D. A PSO-DV based approach for capacitor placement in radial distribution systems. In: Proc. of 2011 24th Canadian Conference on Electrical and Computer Engineering (CCECE). 